\title{
MUSCLE X-RAY ATTENUATION IS NOT DECREASED DURING EXPERIMENTAL BED REST
}

\author{
JÖRN RITTWEGER, MD, ${ }^{1}$ KLAUS MÜLLER, PhD, ${ }^{1}$ MARIE-PIERRE BAREILLE, PhD, ${ }^{2}$ DIETER FELSENBERG, $M D,{ }^{3}$ \\ and JOCHEN ZANGE, PhD ${ }^{1}$ \\ ${ }^{1}$ Institute of Aerospace Medicine, German Aerospace Center, Cologne, Germany \\ ${ }^{2}$ MEDES-IMPS, Clinique Spatiale, Toulouse, France \\ ${ }^{3}$ Center for Muscle and Bone Research, Charité - University Medicine Berlin, Germany \\ Accepted 19 August 2012
}

ABSTRACT: Introduction: Muscle X-ray attenuation (MXA) in Hounsfield units $(H U)$ is decreased in dystrophy, aging, and immobilization. We hypothesized a decrease also during bed rest (BR). Methods: Twenty-five healthy men performed BR for 90 days, 8 of whom performed flywheel exercise 2-3 times per week. Muscle cross-sectional area (CSA) and MXA were assessed by computed tomography. Results: In the control group, calf muscle CSA decreased during 89-day BR by 26.6 (SD 3.8$) \%$, while MXA increased by $1.2 \mathrm{HU}$ on day 28 and almost reached baseline values on day 89 . Changes were mitigated in the flywheel group for the calf $(P<0.001)$. All changes were reversed within 90 days after BR. Conclusions: Results contradict the initial hypothesis. Increased intramuscular blood content, interstitial fluid space shrinkage, and reduction of intramuscular lipid stores are possible explanations for the moderate increase in MXA during the initial phase of bed rest.

Immobilization, clinical conditions, aging, and spaceflight are characterized by reductions in muscle size. ${ }^{1,2}$ In addition, alteration of muscle composition is another emerging feature in muscle atrophy and dystrophy. In Duchenne muscular dystrophy, for example, there is inter- and intramuscular fatty infiltration, ${ }^{3}$ and in the elderly, the term "myosteatosis" has been coined to describe the intramuscular accumulation of lipids. ${ }^{4}$ Such alterations in muscle composition can be assessed by computed tomography as muscle X-ray attenuation (MXA). Results are typically represented in Hounsfield units (HU), where tissue attenuation is expressed in relation to attenuation in water:

$$
M X A[H U]=1000 . \frac{\mu_{\text {Tissue }}-\mu_{H 20}}{\mu_{H 20}}
$$

In practice, muscle tissue has values around 50 $\mathrm{HU}$, and adipose tissue yields negative $\mathrm{HU}$ values as low as $-100 \mathrm{HU}$, depending on the scanner properties. Hence, MXA is negatively correlated with muscle fiber lipid content, ${ }^{5}$ and MXA is reduced in patients with Duchenne dystrophy ${ }^{6}$ and other types of dystrophic and inflammatory muscle

Abbreviations: BDC, baseline data collection; CSA, cross-sectional area; Ctrl, control group; FW, flywheel group; HU, Hounsfield units; MXA, muscle X-ray absorption

Key words: blood content, human physiology, immobilization, physical medicine, peripheral quantitative computed tomography

Correspondence to: J. Rittweger; e-mail: joern.rittweger@dlr.de

(C) 2012 Wiley Periodicals, Inc.

Published online 2 October 2012 in Wiley Online Library (wileyonlinelibrary. com). DOI 10.1002/mus.23644 disorders. ${ }^{7}$ These muscle disorders have a uniform disease-related pattern, ${ }^{7,8}$ while neurogenic muscle atrophy is characterized by anatomically specific fat infiltration in the sub-lesional regions. ${ }^{7}$ Both clinical disorders are concordant, however, in showing negative HU values for muscle.

MXA also decreases as a function of age,$^{9-11}$ although the reduction seems to be more than 10 times smaller than in dystrophy and neurogenic atrophy. Of note, MXA can explain variation in muscle strength ${ }^{11}$ and locomotion performance ${ }^{12}$ in the elderly. Strength training leads to concomitant changes in MXA and muscle size in elderly women, ${ }^{13}$ and de-training and re-training produce MXA effects in very much the same sense. ${ }^{4}$ Moreover, reduced MXA in the elderly can predict falls and fractures. ${ }^{14}$ These latter studies indicate that MXA could serve as an independent, objective indicator of muscle function.

One might expect on the basis of existing literature that MXA should decrease in response to immobilization. Indeed, Berg et al. reported an MXA decrease in the vastus lateralis muscle after 4 weeks of immobilization by unilateral lower limb suspension (ULLS). ${ }^{15}$ In apparent contrast to this, Desplanches et al. reported a $42 \%$ decrease in the volume density of lipids, as assessed by electron microscopy, in the deltoid muscle after 37 days of bed rest. ${ }^{16}$ It is unclear whether this seeming discrepancy is due to anatomical (arm vs. leg) or methodological (X-ray vs. electron microscopy) discrepancy with Berg's study.

To the best of our knowledge, no study has yet investigated the effects of bed rest upon MXA. Experimental bed rest, in particular with $-6^{\circ}$ head down tilt (HDT), is a ground-based model to simulate the effects of microgravity, ${ }^{17}$ and it is also of obvious clinical relevance. One should note here that HDT bed rest is associated with fluid shifts, ${ }^{18}$ arising for a great part from the intramuscular fluid space. ${ }^{19}$ HDT and ULLS therefore are discordant at least with regards to fluid redistribution.

Due to its position in the periodic table of elements, potassium $(K)$ has greater X-ray absorption than sodium $(\mathrm{Na})$. This suggests that any alteration in the ratio between intracellular space [rich in $\mathrm{K}$ 
and phosporous $(\mathrm{P})$, mainly as energy-rich phosphates and macromolecules such as DNA] and extracellular space (rich in $\mathrm{Na}$ ) will affect MXA. MRI examinations which show a decrease in the transversal spin relaxion $\left(\mathrm{T}_{2}\right)$ after space flight and bed rest may indicate that the decrease in leg muscle volume may be more strongly affected by losses in free intracellular water than by reductions in muscle protein content. ${ }^{1,20}$ However, it is currently unclear to what extent fluid re-distribution in HDT and ULLS affects the intra- and extra-cellular compartments. Therefore, and based on the findings by Berg et al. ${ }^{15}$ we hypothesized that MXA would be reduced as a result of experimental bed rest. Moreover, we were interested to explore any relationship between individual changes in MXA and changes in muscle cross-section.

\section{METHODS}

The Long Term Bed Rest (LTBR) study ${ }^{21}$ was designed to scrutinize 2 proposed countermeasures. First, the efficacy of pamidronate, a bone anti-resorptive drug, to prevent HDT-induced bone loss was tested. ${ }^{22}$ Second, flywheel resistive exercise (see below) was tested as a countermeasure against muscle atrophy and bone loss.

Twenty-five healthy male subjects were recruited based on predefined inclusion and exclusion criteria. ${ }^{22}$ Complete lack of physical activity and participation in competitive sports were exclusion criteria, so that the cohort studied can be considered as moderately active. Subjects were randomly assigned to 1 of 3 groups: a group that did bed-rest only $(N=9)$, a group that received a single infusion of $60 \mathrm{mg}$ i.v. pamidronate before bed rest $(N=7)$, or a group $(N=9)$ that performed flywheel training.

Baseline data collection started 2 weeks before the onset of bed rest. Participants underwent 90 days of strict bed rest, and adherence to the protocol was ascertained by closed circuit television plus force transducers that were integrated into the bed mattresses. After reambulation, the participants spent 14 more days in the MEDES facilities for early recovery measurements. ${ }^{23}$ During this entire period, an isocaloric diet was administered, ${ }^{21}$ and body weight was assessed every morning with an Arjo scale with integrated lift trolley (Arjo Bolero CEB 6000-01, ARJO Hospital Equipment AB, Eslov, Sweden). This scale has an accuracy of $\pm 50 \mathrm{~g}$.

Follow up visits were scheduled at 45, 90, 180, and 360 days after re-ambulation. The study was approved by the local Ethics Committee, and all participants gave their written informed consent before inclusion in the study.
Flywheel Training. Resistance exercise was performed during the HDT phase with a "flywheel", i.e. a device that is gravity independent and uses the inertia of a rotating flywheel. ${ }^{24}$ Training sessions were scheduled 2 to 3 times per week. During each session, 4 sets of supine squat (aiming at the knee and hip extensors) and calf press (aiming at the ankle plantarflexors) exercises were performed. Each set consisted of 7 maximum contractions (concentric and eccentric) for the supine squat and 14 maximum contractions for the calf press. Calf press exercise was performed with the forefoot on the footplate, and the heel was free. Between sets, 2 min were given for rest, and $5 \mathrm{~min}$ were allowed between exercises.

pQCT Scans. Muscle scans were obtained by peripheral computed tomography (pQCT), using an XCT2000 scanner (Stratec Medizintechnik, Pforzheim, Germany) as described previously. ${ }^{25}$ The XCT2000 device is dedicated to measure "volumetric bone mineral density". More precisely, it is calibrated to assess hydroxyl apatite density with olive oil as reference $=0$. The scanner's tube voltage was set to $58 \mathrm{kV}$, and the beam width was $2.2 \mathrm{~mm}$. Cross-sectional images were obtained in the horizontal plane at two-thirds of the tibial and the ulnar lengths for the gross calf and forearem crosssections, respectively. These measurement sites were chosen because they coincide with greatest muscle cross-section.

Scans were performed twice during baseline data collection (BDC), namely 14 and 7 days before bed rest commencement, and on the 28th and on the 89th days of bed rest (HDT28 and HDT89, respectively), as well as $14,90,180$, and 360 days after re-ambulation $(\mathrm{R}+14, \mathrm{R}+90, \mathrm{R}+180$, and $\mathrm{R}+360$, respectively). Results from the 2 baseline scans were lumped together to yield a single BDC value and also to assess the reproducibility of measurements.

Blood Samples. During the bed rest study, routine blood samples were obtained during BDC-14 and on days HDT14, HDT30, HDT60, HDT90, R+13, and $\mathrm{R}+45$ and analyzed for hemoglobin concentration $(\mathrm{Hb})$. These data are included here as a surrogate measure for the hemoconcentration effect associated with bed rest and the recovery from it. $^{26,27}$

Phantom Measurements. To assess machine-specific X-ray attenuation of the relevant chemical elements (Ca, Cl, Fe, K, Na, P, S), solutions were prepared in graded concentrations. Soluble compounds were chosen in which the counter ion was of low chemical order (Ca-nitrate, ammonium chloride, ferrous sulphate, lime potash, Na-acetate, 
Na-phosphate, sulphuric acid). These solutions were prepared in concentrations of 50, 100, 200, 400, and $1000 \mathrm{mM}$ (exceptions: ferrous sulphate concentrations were 5, 25, 50, 100, and $250 \mathrm{mM}$ ). The range of concentrations used was in excess of the expected range for physiological variation to arrive at robust estimates. Moreover, a blood sample was drawn for an additional phantom and diluted 1:1 and 1:3 in distilled water. These phantoms were then stored in plastic containers and scanned with the XCT device with the same scan parameters as for the muscle scans.

Data Processing. Visual inspection of the muscle scans suggested that bed rest did not result in inter-muscular lipid accumulation. Hence, a decision was made to consider MXA within the entire anatomical outline of the muscle. To this end, regions of interest (ROI) were placed so that the gross muscle cross-section was included by setting the ROI exactly on the limit between muscle and fat and between muscle and the tibia (or ulna in the forearm), respectively. To suppress a bias by the partial volume effect, 2 layers of pixels were peeled off by pressing the "cursor-down" function in the XCT software twice so that the analyzed ROI had its entirety within the muscle. Next, another ROI was placed on the limit between muscle and the fibula (radius in the forearm), and then increased by 2 pixels so that the ROI to be excluded was entirely within the muscle. Pixel hydroxyl apatite density (PHAD) was computed for the muscle area after subtraction of the fibula/ radius ROI. X-ray attenuation $(\mu)$ was then computed from PHAD values as:

$$
\mu=\frac{P H A D-x \text { Inter }}{x \text { Slope }}
$$

where $x$ Inter and xSlope are the calibration parameters specific for each XCT scanner type with specific values of $322 \mathrm{mg} \cdot \mathrm{cm}^{-3}$ and $1724 \mathrm{mg}^{-1} \cdot \mathrm{cm}^{4}$ in our case. The attenuation coefficient of water turned out to be $0.220 \mathrm{~cm}^{-1}$ for our scanner.

Finally, changes from BDC were computed for days HDT28, HDT89, and $\mathrm{R}+14$ for muscle crosssection $\left(\Delta \mathrm{mCSA}\right.$, in $\left.\mathrm{cm}^{2}\right)$ as well as for muscle X-ray absorption ( $\triangle \mathrm{MXA}$, in $\mathrm{HU})$.

Statistical Analysis. Reproducibility of MXA and CSA was assessed as the short term error, i.e., the standard deviation between the 2 baseline measurements on BDC-14 and BDC-7. ${ }^{28}$ Effects of bed rest and flywheel training were assessed with linear mixed effect (LME) models that were generated for MXA, CSA, and $\mathrm{Hb}$ values, with time and group as fixed effects and subject ID as random effect. Variances were allowed to differ between participants and group, and LME models were optimized according to the Akaike information criterion (see p. 353 and p. 652 in Crawley ${ }^{29}$ ). Correlation and regression analysis were carried out after visual inspection of the data to explore the relationship between $\triangle \mathrm{mCSA}, \triangle \mathrm{MXA}$ and $\mathrm{Hb}$ values. Correlation analysis was likewise performed for values obtained on days HDT89 and $\mathrm{R}+14$. This was done separately for $\triangle \mathrm{mCSA}$ and $\triangle \mathrm{MXA}$ to explore how far MXA changes reflect individual characteristic information persisting through the re-ambulation phase. Differences in anthropometric variables between groups were tested by the Student $t$-test. Phantom measurements were analyzed by linear correlation and regression for the relationship between concentration and X-ray attenuation.

Statistical analyses were carried out in the "R" statistical environment (version 2.9.2, www.r-project. org). Post hoc power analyses were performed, where desired, using the $\mathrm{G}^{*}$ Power software in its version 3.1.2 (www.psycho.uni-duesseldorf.de/aap/projects/ gpower). Data are given as means and standard deviations (SD) if not indicated otherwise. The level for statistical significance was set to $\alpha=0.05$.

\section{RESULTS}

During the study, 1 participant (K2) in the flywheel group (FW) group had to discontinue the flywheel training during the second half of the bed rest phase, which made an assignment to either of the groups impossible. Data of this participant have been excluded from this publication accordingly. As anticipated, pamidronate had no effect on muscle, ${ }^{23}$ and data from the control group and the group that received pamidronate were therefore lumped together into 1 control group (Ctrl) and compared with the FW. Baseline anthropometric data were comparable between the Ctrl and FW groups, as can be seen from Table 1 .

The short term error was $130 \mathrm{~mm}^{2}$ [coefficient of variation $(\mathrm{CV})=1.8 \%$ ] for calf $\mathrm{mCSA}, 1.5 \mathrm{HU}$ $(\mathrm{CV}=2.9 \%)$ for calf $\mathrm{MXA}, 76 \mathrm{~mm}^{2}(\mathrm{CV}=2.0 \%)$ for forearm $\mathrm{mCSA}$, and $1.4 \mathrm{HU}(\mathrm{CV}=2.5 \%)$ for forearm MXA.

Calf Muscle Changes Over Time. Statistical analysis of calf mCSA revealed significant effects of time

Table 1. Anthropometric characteristics at baseline.

\begin{tabular}{lccccc}
\hline & $N$ & $\begin{array}{c}\text { Age } \\
{[\text { years] }}\end{array}$ & $\begin{array}{c}\text { Height } \\
{[\mathrm{cm}]}\end{array}$ & $\begin{array}{c}\text { Weight } \\
{[\mathrm{kg}]}\end{array}$ & $\begin{array}{c}\mathrm{BMl} \\
{\left[\mathrm{kg} / \mathrm{m}^{2}\right]}\end{array}$ \\
\hline Ctrl & 16 & 32.5 & 174.2 & 71.4 & 23.5 \\
& \multirow{3}{*}{ FW } & $(3.4)$ & $(3.9)$ & $(6.7)$ & $(1.7)$ \\
& 8 & 30.8 & 175.5 & 71.0 & 23.1 \\
P-value & & $(5.8)$ & $(4.9)$ & $(5.7)$ & $(1.6)$ \\
\hline
\end{tabular}

Data are listed as mean values (and SD in brackets). 

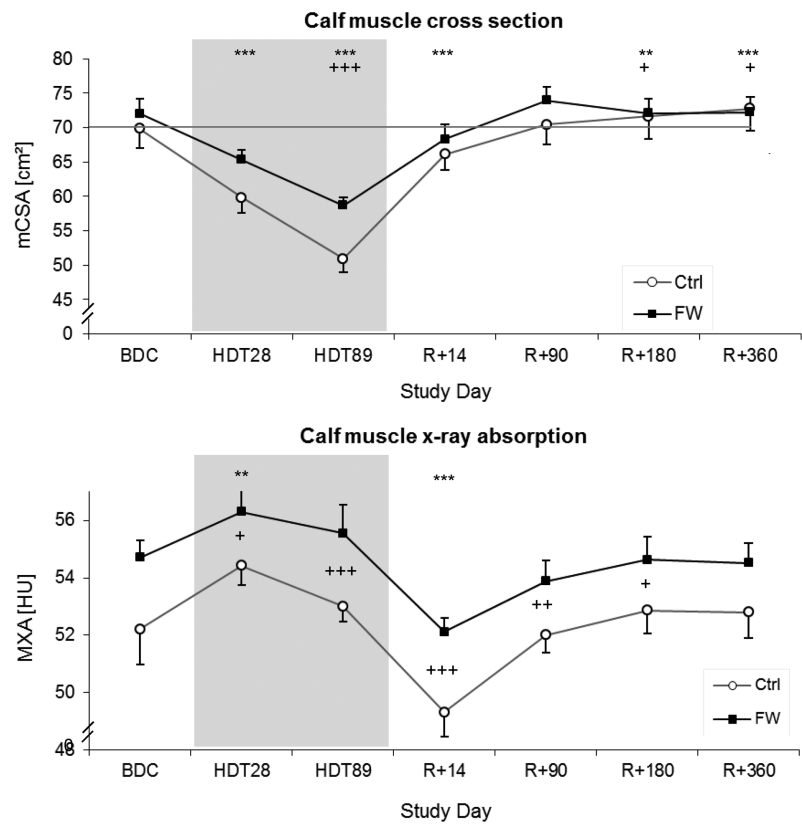

FIGURE 1. Calf muscle cross-sectional area (mCSA, upper panel) and X-ray absorption (MXA, lower pane), expressed in Hounsfield units $(\mathrm{HU})$, for the control group (Ctrl) and the group that did resistive flywheel exercise during 90 days of bed rest. Data are displayed for baseline data collection (BDC), for days 28 and 89 of bed rest (HDT28 and HDT89, respectively), and $14,90,180$, and 360 days following re-ambulation $(R+14$, $\mathrm{R}+90, \quad \mathrm{R}+180$, and $\mathrm{R}+360$, respectively). Calf mCSA decreased during bed rest, but super-recovery was observed 180 and 360 days after re-ambulation. Both effects were more pronounced in Ctrl than in FW. For MXA, a small but significant increase over baseline was observed during the bed rest phase (HDT28 and HDT89), whereas values were decreased 14 days following re-ambulation $(R+14)$. Again, these changes were more pronounced in the $\mathrm{Crl}$ than in the FW group. No further change was observed during the follow-up visits on days 90 , 180 , or 360 after re-ambulation. Data are means and their standard errors. Time effects: ${ }^{* \star *} P<0.001,{ }^{* \star} P<0.01$; Time ${ }^{*}$ group interaction: ${ }^{+++} P<0.001,{ }^{++} P<0.01$.

and a time * group interaction $(P<0.001$ in both cases), but not for group ( $P=0.29$, see Fig. 1$)$. The time effect indicated significant reductions in calf mCSA on days HDT28, HDT89, and $\mathrm{R}+14$ $(P<0.001$ in all instances $)$, but significant increases over baseline on days $\mathrm{R}+180$ and $\mathrm{R}+360$ $(P=0.001$ and $P<0.001$, respectively). The decrement in calf mCSA was smaller in the FW $(-18.6$ $\%$, SD $3.0 \%$ ) group compared with the Ctrl group $(-26.6 \%$, SD $3.8 \%)$ on day HDT89 $(P<0.001)$, and so was the increase over baseline values on day $\mathrm{R}+180(-0.2 \%$ vs. $3.8 \%, P=0.046)$ and on day $\mathrm{R}+360(0.8 \%$ vs. $4.3 \%, P=0.037)$, indicating that the "super-recovery" cleared away and thus was limited in time in the FW group.

For calf MXA, a main effect was found for time as well as a time * group interaction $(P<0.001$ in both cases, see Fig. 1.A), but no main effect for group $(P=0.086)$. MXA increased between BDC and day HDT28 $(P=0.007)$. The increase amounted to $1.2 \mathrm{HU}$ (SE 0.44) in FW, and to 2.1 HU (SE 0.46) in Ctrl ( $P$ for time * group effect $=$ $0.045)$. On day HDT89, MXA was increased in Ctrl only $(P<0.001)$. Conversely, a pronounced decrease by -3.7 (SE 0.44) HU was observed on day $\mathrm{R}+14$ compared with baseline values $(P<$ $0.001)$. This change was more pronounced in Ctrl than in FW $(P<0.001)$, and the Ctrl group depicted greater increases during follow up on days $\mathrm{R}+90$ and $\mathrm{R}+180(P=0.005$ and $P=0.019$, respectively).

Forearm Muscle Changes Over Time. Forearm mCSA yielded a significant main effect for time $(P<$ $0.001)$, but no group effect or group * time interaction was found $(P=0.72$ and $P=0.75$, respectively, see Fig. 2). Accordingly, forearm mCSA was reduced by 1.8 (SE 0.4$) \mathrm{cm}^{2}$ on day HDT28, by 2.8 (SE 0.4$) \mathrm{cm}^{2}$ on HDT89, and by $2.5(\mathrm{SE} 0.4) \mathrm{cm}^{2}$ on $\mathrm{R}+14(P<0.001$ in all instances $)$.

For forearm $M X A$, significant group and time effects were found $(P<0.001$ in both cases $)$, but no group $*$ time interaction $(P=0.53)$. However, the magnitude of these effects was small, amounting to a group difference by 0.5 (SE 0.86) HU, and the time effect was isolated to an increase by 1.7 (SE 0.6$) \mathrm{HU}$ on day $\mathrm{R}+90(P=0.006)$.
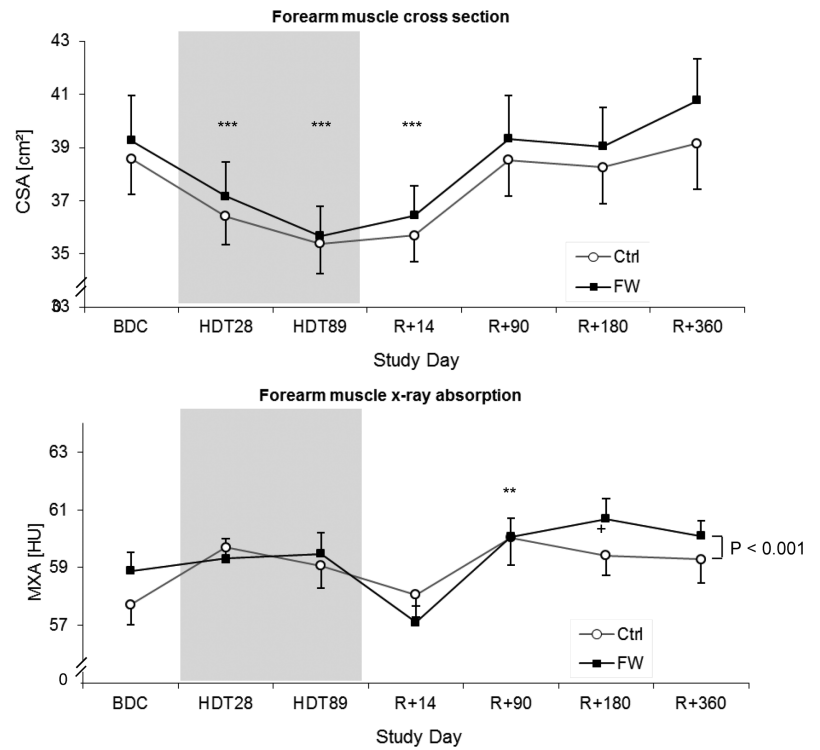

FIGURE 2. Forearm mCSA (upper pane) and MXA (lower pane). Similar to calf $\mathrm{mCSA}$ in Figure 1, forearm mCSA decreased during bed rest. However, there was no group difference, and changes in forearm mCSA were much smaller than those in calf mCSA. For forearm MXA, statistical analyses yielded group and time effects $(P<0.001)$, but no group*time interaction. Follow-up tests showed that this group*time interaction was limited to $R+90$, which seems to be a somewhat inconsistent finding. Data are means and their standard errors. $P<0.001$; Time effects: ${ }^{\star \star *} P<0.001,{ }^{\star *}: P<0.01$. 
Table 2. Correlation matrix for changes at the end of bed rest.

\begin{tabular}{|c|c|c|c|c|}
\hline & $\begin{array}{c}\text { Forearm } \\
\Delta \mathrm{mCSA}\end{array}$ & $\begin{array}{c}\text { Calf } \\
\Delta \mathrm{MXA}\end{array}$ & $\begin{array}{c}\text { Forearm } \\
\Delta \mathrm{MXA}\end{array}$ & $\Delta \mathrm{Hb}$ \\
\hline $\begin{array}{l}\text { Calf } \Delta \mathrm{mCSA} \\
\text { Forearm } \Delta \mathrm{mCSA} \\
\text { Calf } \Delta \mathrm{MXA} \\
\text { Forearm } \Delta \mathrm{MXA}\end{array}$ & n.s. & $\begin{array}{l}\text { n.s. } \\
-0.42^{\star}\end{array}$ & $\begin{array}{l}-0.46^{\star} \\
\text { n.s. } \\
0.45^{\star}\end{array}$ & $\begin{array}{l}\text { n.s. } \\
\text { n.s. } \\
\text { n.s. } \\
\text { n.s. }\end{array}$ \\
\hline
\end{tabular}

Changes between baseline and the end of bed rest for calf muscle cross-section, calf muscle X-ray attenuation, forearm muscle crosssection, forearm muscle $X$-ray attenuation, and hemoglobin. This table thus highlights the commonality of responses to bed rest in these variables.

${ }^{*} P<0.05$.

Blood Hemoglobin Concentration. No group * time interaction was found for blood hemoglobin concentration ( $P$-values 0.12 and 0.95 ), and we report averages across groups here. Hemoglobin values increased from $146(\mathrm{SD} 7.0) \mathrm{g} / \mathrm{L}$ by $6.9 \mathrm{~g} / \mathrm{L}(\mathrm{SE}=$ 1.3 for all changes from baseline) $\mathrm{g} / \mathrm{L}$ on day HDT14 $(P<0.001)$ and by $6.7 \mathrm{~g} / \mathrm{L}$ on day HDT60. Conversely, values were decreased below BDC levels on day $\mathrm{R}+13$ by $12.0 \mathrm{~g} / \mathrm{L}(P<0.001)$ and by $3.6 \mathrm{~g} / \mathrm{dl}$ on day $\mathrm{R}+45(P=0.067)$.

Inter-relationship of Changes. The correlation matrix for changes between baseline and the end of bed rest is given in Table 2. Significant correlations were found between calf $\triangle \mathrm{MXA}$ and forearm $\Delta \mathrm{mCSA} \quad(P=0.038)$, between calf $\Delta \mathrm{mCSA}$ and forearm $\triangle \mathrm{MXA}(P=0.019)$, and between calf $\triangle \mathrm{MXA}$ and forearm $\triangle \mathrm{MXA}(P=0.025)$. Correlation analyses between $\triangle \mathrm{mCSA}$ and $\triangle \mathrm{MXA}$ within the calf and within the forearm revealed negative $r$-values, but failed to achieve statistical significance ( $P=0.086$ for the calf and $P=0.73$ for the forearm). Of note, $\Delta \mathrm{Hb}$ was unrelated to any of the other variables $(P>0.59$ in all cases $)$.

By contrast, highly significant correlations were found when changes at the end of bed rest (HDT89) were compared with changes after 2 weeks recovery, except for calf $\triangle \mathrm{mCSA}$ (see Table 3). This suggests that individual MXA-

Table 3. Correlation coefficients for changes at the end of bed rest and after 2 weeks of recovery.

\begin{tabular}{ll}
\hline & \multicolumn{1}{c}{$r$} \\
\hline$\Delta$ mCSA Calf & $n . s$. \\
$\Delta$ mCSA Forearm & $0.94^{\star \star \star}$ \\
$\Delta$ MXA Calf & $0.67^{\star \star \star}$ \\
$\Delta$ MXA Forearm & $0.53^{\star \star}$ \\
$\Delta \mathrm{Hb}$ & $0.72^{\star \star \star}$ \\
\hline
\end{tabular}

The correlation between changes at the end of bed rest and after 2 weeks of recovery for calf muscle cross-section, calf muscle $X$-ray attenuation, forearm muscle cross-section, forearm muscle $X$-ray attenuation, and hemoglobin. This table thus highlights the persistence of bed rest-induced changes during early recovery.

${ }^{* *} P<0.01$.

${ }^{* * *} P<0.001$.
Table 4. Results of phantom measurements.

\begin{tabular}{|c|c|c|c|c|c|c|c|}
\hline Element & Compound & $\begin{array}{c}\text { Slope } \\
{[\mathrm{HU} / \mathrm{mM}]}\end{array}$ & $\begin{array}{c}\mathrm{SE} \\
{[\mathrm{HU} / \mathrm{mM}]}\end{array}$ & $\begin{array}{l}\mathrm{C}_{\mathrm{IC}} \\
{[\mathrm{mM}]}\end{array}$ & $\begin{array}{l}X A_{I C} \\
{[H U]}\end{array}$ & $\begin{array}{l}\mathrm{C}_{\mathrm{EC}} \\
{[\mathrm{mM}]}\end{array}$ & $\begin{array}{l}X A_{E C} \\
{[H U]}\end{array}$ \\
\hline $\mathrm{Fe}$ & $\mathrm{FeSO}_{4} \cdot 7 \mathrm{H}_{2} \mathrm{O}$ & 1.123 & 0.012 & 0.1 & 0.1 & 0 & 0 \\
\hline $\mathrm{Ca}$ & Ca-Nitrate & 0.323 & 0.002 & 2 & 0.6 & 5 & 1.6 \\
\hline K & $\mathrm{KOH}$ & 0.182 & 0.004 & 145 & 26.4 & 4 & 0.7 \\
\hline$P$ & $\mathrm{NaH}_{2} \mathrm{PO}_{4} \cdot \mathrm{H}_{2} \mathrm{O}$ & 0.145 & 0.003 & 100 & 9.6 & 2 & 0.2 \\
\hline S & $\mathrm{H}_{2} \mathrm{SO}_{4}$ & 0.139 & 0.002 & 20 & 2.8 & 1 & 0.1 \\
\hline $\mathrm{Cl}$ & $\mathrm{NH}_{4} \mathrm{Cl}$ & 0.112 & 0.004 & 3 & 0.3 & 114 & 12.8 \\
\hline $\mathrm{Na}$ & $\mathrm{NaAc}$ & 0.049 & 0.003 & 10 & 0.7 & 145 & 7.1 \\
\hline
\end{tabular}

The slope of the regression equation and its standard error (SE) and the concentrations $(C)$ and in the intra- and extracellular space, respectively (values are taken from http://physioweb.uvm.edu/bodyfluids/ionic.htm). From these, the $X$-ray attenuation $(X A)$ is calculated as $X A=$ slope.C. For computing $X A$ values for $P$, the Na-slope was subtracted, and the $S$-slope was subtracted for computing XA for Fe. Elements have been ordered by the slope. For all regressions, $P<0.001$ and $R^{2}>0.95$ (except for $\mathrm{Na}$ acetate, where $R^{2}=0.86$ ).

changes as well as forearm mCSA changes, but not calf mCSA changes, were persistent through the re-ambulation phase.

Phantom measurements. The X-ray attenuation characteristics of the various elements tested are given in Table 4. Of those tested, Fe had the strongest attenuation properties, and $\mathrm{Na}$ had the weakest, as can be seen from the column "slope" in the Table. Moreover, the table gives the contribution of each ion to the X-ray attenuation within the intracellular and extracellular compartments. Lumping these together, a sum of $40.3 \mathrm{HU}$ and 22.5 HU can be derived for the ionic contribution to the intra- and extracellular X-ray attenuation, respectively.

The scanned blood sample had a hemoglobin concentration of $153 \mathrm{~g} / \mathrm{L}$, a hematocrit of $44.1 \%$, and a serum $\mathrm{Fe}$ concentration of $15.5 \mu \mathrm{M}$; it yielded a slope of 0.72 (SE 0.030) HU per \% dilution.

\section{DISCUSSION}

We undertook this retrospective analysis to explore whether experimental bed rest elicits alterations in the X-ray attenuation of skeletal muscle, and to generate hypotheses about the possible mechanisms behind such changes. Along with the well documented atrophy of the calf musculature, and with the somewhat unexpected decrease in forearm muscle size, MXA in the calf muscles was found to not decrease, but to even increase during bed rest (Fig. 1, lower pane), and to dip during early recovery. It should be considered, though, that the increase was only moderate, and that the between-group variation almost reached statistical significance $(P=0.086)$. On the other hand, the observed changes appeared quite systematic, and very recent data suggest that MXA, as assessed with the same device as in this study can predict fragility fracture in older women, ${ }^{30}$ which suggests that 
the methodology applied in this study assesses meaningful information.

Of interest, the time course of changes in the forearm (Fig. 2) appeared to be quite similar to those in the calf, although on a smaller and not significant scale. It is also interesting to note that the time course was discordant between MCSA and MXA changes, both for the calf and the forearm: While mCSA depicted a simple pattern of loss and recovery, the MXA pattern was "bi-phasic," with a sharp dip during early recovery.

The finding of an increased MXA seems to be compatible with the decreased lipid droplet density after bed rest reported by Desplanches et al. ${ }^{16}$ but it is at variance with the reported MXA decrease reported by Berg et al. ${ }^{15}$ Of note, the latter study differs from the present not only in terms of duration (28 days vs. 90 days) and type of immobilization (limb-suspension vs. bed rest) but also in the anatomical site of MXA measurement (thigh vs. calf) and methodology (scanner type). The fact that MXA changes in this study were consistent after 4 weeks and after 90 days discards time as explanatory factor. For the sake of brevity and parsimony, we will also not further discuss site-effects and thus concentrate on model (i.e., alignment of the main body axis) and measurement (i.e., X-ray energy) as possible confounders between the 2 studies.

Are $X$-ray attenuation changes related to changes in Fe-concentration? As demonstrated in Table $4, \mathrm{Fe}$ is a potent X-ray absorber. Of all the $F e$ in the body, approximately $75 \%$ is within the blood, the largest fraction of which is bound to hemoglobin, and minor fractions are bound to ferritin, transferrin, or are nonenzymatically bound. ${ }^{31}$ It is also well known that total blood volume decreases during bed rest, predominantly due to plasma losses with a relative sparing of the blood cell fraction. ${ }^{32}$ This so-called hemoconcentration peaks during the initial phase of bed rest. This is confirmed by Figure 3, in which $\mathrm{Hb}$ depicts a time course that is very similar to the MXA changes in Figures 1 and 2. Hence, could hemoconcentration explain the MXA changes?

The lack of any correlation between individual changes in $\mathrm{Hb}$ and MXA in Table 2 contradicts this idea. Moreover, one has to consider that the effective concentration of blood in the resting vastus lateralis muscle is only $4 \%$ of its volume. ${ }^{33}$ Hence, an increase in hemoconcentration by $2 \%$ will lead to an increase in muscular $\mathrm{Hb}$ concentration of less than 1\%. Based on our blood phantom measurements (see last section of results), the hemoconcentration observed in this study can

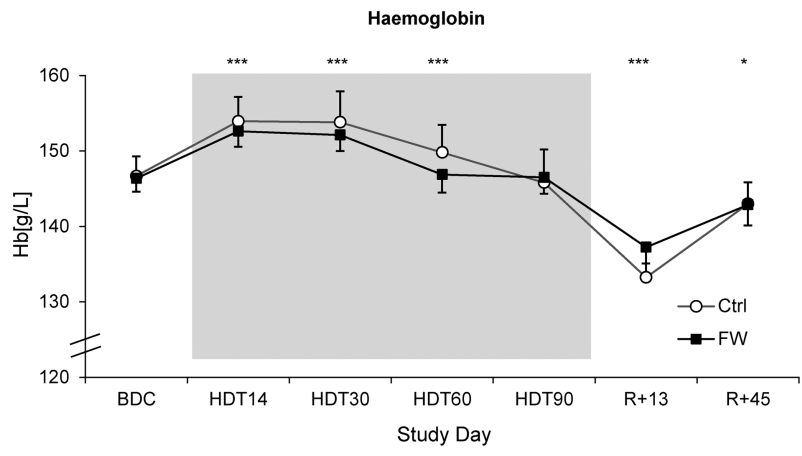

FIGURE 3. Hemoglobin $(\mathrm{Hb})$ concentration over time. Similar to calf mXA in the calf (Fig. 1) and the forearm (Fig. 2), there is an increase during the bed rest phase and a dip during early recovery. Data are means and their standard errors. Time effects: ${ }^{* \star *} P<0.001,{ }^{\star} P<0.05$. No significant time ${ }^{*}$ group interaction was found ( $P$-values between 0.12 and 0.95$)$.

account for an increase in MXA by less than 0.1 $\mathrm{HU}$, i.e., only a fraction of the observed change.

It is well established that $-6^{\circ}$ head down tilt enhances venous return from the legs, and blood (and $\mathrm{Fe}$ ) concentration within the calf musculature should therefore decrease during HDT. However, we expect this effect to be rather small, because subcutaneous veins were excluded in the image analysis, and because the subject had been in the supine position for $1 \mathrm{~h}$ before scanning for BDC and recovery sessions to preclude a bias by blood volume shifts. ${ }^{19}$ Instead, there is evidence to assume increased blood concentration in immobilized muscle, as for example, nerve compression in the rat has been demonstrated to increase blood concentration in resting muscle by 3-fold. ${ }^{34}$ This effect may be due partly to relative sparing of capillaries during immobilization-induced atrophy. ${ }^{35}$ This opens the possibility that tilt-induced headward blood volume shifts may have been counteracted by immobilization-induced blood pooling in the calf muscle (the proposition of increased blood concentration is made notwithstanding the well-established finding of reduction in skeletal muscle perfusion by immobilization $\left.{ }^{36}\right)$. Using the same numerical figures as above $[1 \mathrm{HU} /(4 \%$. $(0.72-0.54) \mathrm{HU} / \%)]$, we see that a 2.4-fold increase in muscular blood content can explain an MXA increase by $1 \mathrm{HU}$ during the bed rest phase. Thus, blood content is a possible contributor. However, the lack of a clear correlation between individual changes in MXA and mCSA $(P=0.086$ for the calf) may speak against it, and so probably does the discordant time course of those 2 variables.

There is, of course, also a remote possibility to link the observed MXA changes to changes in myoglobin concentration, provided there was a relative sparing of myoglobin by the atrophy process. However, myoglobin concentrations in skeletal muscle 
are very low $(100 \mu \mathrm{M})$, and the lack of correlation between changes in MXA and in mCSA may speak against this possibility.

Possible Effects of Electrolyte Changes. Even though the MXA changes observed in this study could be explained by accumulation of $F e$, in principle this seems unlikely to explain fully the discrepancy of MXA changes between this study and the original ULLS study. ${ }^{15}$ As discussed above, HDT and ULLS are fundamentally different with regard to the gravity vector. Moreover, there is another discrepancy between this study, where forearm muscle size was decreased during $-6^{\circ}$ HDT bed rest, and the Berlin (horizontal) Bed Rest (BBR) study, where no such change was found. ${ }^{37}$ We would argue that subjects in both studies were maintaining the habitual usage of their hands and arms during bed rest, and we are accordingly denying genuine muscle atrophy (i.e., reduction in myofibrillar protein) in the arms in either study. Considering also that the lower arm is below the level of hydrostatic indifference in humans, we also expect position-dependent fluid shifts from the forearm, albeit on a smaller scale than from the calf. Hence, bearing the similarity of the time courses for the calf and the forearm in mind (upper panels in Figs. 1 and 2) it is tempting to ascribe the reduction in forearm mCSA to such fluid shifts. Is it also possible to explain the MXA increase in the forearm muscle on day $\mathrm{R}+90$, and perhaps also in the calf muscle in terms of fluid re-distribution?

One way for this to happen would be an increase of the K:Na-ratio due to increases in intrato extracellular space within the tissue. Summing up the main constituents that cause X-ray attenuation, we arrive at an estimate of 40.3 HU for X-ray attenuation in the intracellular space and 22.5 HU for the extracellular space (see Table 4). Based on these figures, one has to demand that the extracellular space shrink by $1 /(40.3-22.5)=5.6 \%$ more than the intracellular space to explain an increase in MXA by $1 \mathrm{HU}$. Although the relative re-distribution of intracellular and interstitial fluid spaces in response to bed rest is not well established, it is well known that $K$ leaks from the skeletal muscle cell into the interstitial space after acute hemorrhage, ${ }^{38}$ from where it is subsequently removed. Importantly, the stimulus for these changes is poor filling of the vessels, and the shifts must therefore be expected to be greater in the interstitial as compared to the intra-cellular compartment. It seems therefore likely that a similar process will occur in the more chronic redistribution in bed rest and that the interstitial space may shrink more markedly than the intracellular fluid space. Therefore, and despite the fact that we lack precise information on the relative shrinkage of the extra- vs. intracellular space, we believe that this could be a possible mechanism to explain the observed MXA changes in this study. However, one would expect that redistribution of fluid spaces, as well as hemoconcentration, but not in capillary density, should be normalized after 14 days of recovery. In this context, the results of Table 3, i.e., persistent individual MXA changes as revealed by the correlation between changes on days HDT89 and $\mathrm{R}+14$, speak against extra-cellular space shrinkage as the sole driver.

Another element that could be thought of in this context is phosphorus, the concentration of which is on the order of magnitude of $100 \mathrm{mM}$ in muscle and which is present mainly in the form of energy-rich phosphates. However, the concentration of these energy-rich phosphates is not affected by bed rest or space flight, ${ }^{39}$ and we would thus argue that they are unlikely to have contributed to the MXA changes.

\section{Alteration of Intramuscular Lipid Content as a Mecha-}

nism. Finally, we have to come back to the discussion of intramuscular lipid content. Our findings clearly refute the notion that myosteatosis is the determinant of MXA in bed rest, at least in young recreationally active adults. It rather seems that results are compatible with the observation by Desplanches et al. who report a decrease in the volume density of lipids in the deltoid muscle after 37 days of bed rest. ${ }^{16}$ Even though myosteatosis is a hallmark of aging and is associated with deterioration in muscle function, ${ }^{11}$ it is also the case that skeletal muscle of endurance athletes is characterized by high intracellular lipid content ${ }^{40}$ as an adaptation to their specific exercise regimen. ${ }^{41}$ Thus, there seems to be a U-shaped relationship between physical activity and lipid content of muscles. Moreover, intramuscular lipid content is also affected by diet, ${ }^{42,43}$ which has been standardized in this study, but apparently not in the original ULLS study. ${ }^{15}$ Lipid content, therefore, remains a possible, if not likely explanation of the findings made in this study.

Finally, we have to make a technical remark. As can be seen from eq. (1), the Hounsfield unit normalizes X-ray attenuation in relation to water. However, the specific attenuation of a given tissue is also dependant on the energy of the X-ray. Greater anode voltage will generate a "harder beam" that will be less attenuated in relative terms by higher order elements $(K, N a, F e)$ than by adipose tissue. In other words, Hounsfield units are specific to the X-ray spectrum issued by the CT scanner. The scanner in this study was using an 
anode voltage of $58 \mathrm{kV}$ only, and it would be relatively more sensitive to lipid changes than the types of clinical scanners used by Berg et al. ${ }^{15}$ The scanner type used may also explain why MXA values in this study were lower than those reported by Lang et al., ${ }^{14}$ although the fact that MXA values in this study were slightly greater in the forearm than in the calf may suggest that MXA is also different in different muscle groups. Unfortunately, however, there are no adjustments possible to truly compare MXA results across studies.

In the light of the small but consistent alterations induced by bed rest, the most important outcome of this study is the quest for a standardized methodological approach to assess skeletal muscle composition. The basic idea would be to use X-ray based technology to assess lipid content and the intra/extra-cellular fluid ratio. A first step in this direction would be a phantom that includes water, different solutions of elements (analogous to Table 4 ), plus a lipid solution (for the sake of completeness, olive oil yielded a value of $-146 \mathrm{HU}$ on our scanner). In the long run, however, it would be beneficial to have a coherent approach on the measurement approach. One way of doing so could be with dual energy computed tomography (DECT). This may be fruitful in the field of general myology quite as much as for clinical nutrition.

\section{CONCLUSIONS}

In conclusion, X-ray attenuation of the calf muscle did not decrease during bed rest in this study. Of the factors discussed here, we hold 3 as possible explanations, namely (a) increased blood concentration in the muscle tissue, (b) shrinkage of the interstitial fluid space relative to the muscle intracellular space, and (c) reduction of intramuscular lipid stores during bed rest. The lack of coherence in individual changes in muscle size and X-ray attenuation at the same anatomical site, but not in comparison to distant sites (Table 2) may suggest that more than 1 of the 3 explanations mentioned is substantially involved.

On a practical level, we would argue that simple inferences from muscle X-ray attenuation upon myosteatosis can be prone to errors due to fluid distribution. More importantly, the variety of results in the literature relative to MXA calls for standardization of methods.

The Long Term Bed Rest Study was carried out in 2001 and 2002 at the Institut de Médecine et Physiologie Spatiales (MEDES), Toulouse/France and was organized by the European Space Agency and the Japanese Aerospace Development Agency (NASDA, now part of Japanese Aerospace Exploration Agency = JAXA). Our (Rittweger \& Felsenberg) participation in the study was supported by the German Aerospace Center (DLR: $50 \mathrm{WB}$ 0156). We express our gratitude to the staff at MEDES. All of them, but particularly Alain Maillet, and "les deux Pascales" (Cabrole \& Vasseur) have been delightful persons to work with on a daily basis. Per Tesch, Björn Alkner and Jean-Francois Grosset have provided excellent work in providing and maintaining the facilities for the flywheel training. Specific thanks go to Dr. Johannes Willnecker of the Stratec-Medizintechnik company for his advice on the transformation of bone mineral density values into Hounsfield units. Last, but certainly not least, we are indebted to the study participants. Without their selfless contribution, this work would not have been possible. At the time that the LTBR-study was conducted, Jörn Rittweger was funded as a member of the Free University Berlin by the German Aerospace Center (DLR), which is a nonprofit institution that is governmentally funded. He is now (as of November 2009) working full-time for DLR. All authors declare no conflict of interest.

\section{REFERENCES}

1. LeBlanc A, Lin C, Shackelford L, Sinitsyn V, Evans H, Belichenko O, et al. Muscle volume, MRI relaxation times (T2), and body composition after spaceflight. J Appl Physiol 2000;89:2158-2164.

2. Narici MV, Maffulli N. Sarcopenia: characteristics, mechanisms and functional significance. Br Med Bull 2010;95:139-159.

3. Jones DA, Round JM, Edwards RH, Grindwood SR, Tofts PS. Size and composition of the calf and quadriceps muscles in Duchenne muscular dystrophy. A tomographic and histochemical study. J Neurol Sci 1983;60:307-322.

4. Taaffe DR, Henwood TR, Nalls MA, Walker DG, Lang TF, Harris TB. Alterations in muscle attenuation following detraining and retraining in resistance-trained older adults. Gerontology 2009;55:217-223.

5. Goodpaster BH, Kelley DE, Thaete FL, He J, Ross R. Skeletal muscle attenuation determined by computed tomography is associated with skeletal muscle lipid content. J Appl Physiol 2000;89:104-110.

6. Liu M, Chino N, Ishihara T. Muscle damage progression in Duchenne muscular dystrophy evaluated by a new quantitative computed tomography method. Arch Phys Med Rehabil 1993;74:507-514.

7. Nordal HJ, Dietrichson P, Eldevik P, Gronseth K. Fat infiltration, atrophy and hypertrophy of skeletal muscles demonstrated by X-ray computed tomography in neurological patients. Acta Neurol Scand 1988;77:115-122.

8. Kanda F, Fujii Y, Takahashi K, Fujita T. Dual-energy X-ray absorptiometry in neuromuscular diseases. Muscle Nerve 1994;17:431-435.

9. Borkan GA, Hults DE, Gerzof SG, Robbins AH, Silbert CK. Age changes in body composition revealed by computed tomography. J Gerontol 1983;38:673-677.

10. Delmonico MJ, Harris TB, Visser M, Park SW, Conroy MB, Velasquez-Mieyer P, et al. Longitudinal study of muscle strength, quality, and adipose tissue infiltration. Am J Clin Nutr 2009;90:1579-1585.

11. Goodpaster BH, Carlson CL, Visser M, Kelley DE, Scherzinger A, Harris TB, et al. Attenuation of skeletal muscle and strength in the elderly: The Health ABC Study. J Appl Physiol 2001;90:2157-2165.

12. Visser M, Kritchevsky SB, Goodpaster BH, Newman AB, Nevitt M, Stamm E, et al. Leg muscle mass and composition in relation to lower extremity performance in men and women aged 70 to 79 : the health, aging and body composition study. J Am Geriatr Soc 2002; 50:897-904.

13. Sipila S, Suominen H. Effects of strength and endurance training on thigh and leg muscle mass and composition in elderly women. J Appl Physiol 1995;78:334-340.

14. Lang T, Cauley JA, Tylavsky F, Bauer D, Cummings S, Harris TB. Computed tomographic measurements of thigh muscle cross-sectional area and attenuation coefficient predict hip fracture: the health, aging, and body composition study. J Bone Miner Res 2010; 25:513-519.

15. Berg HE, Dudley GA, Haggmark T, Ohlsen H, Tesch PA. Effects of lower limb unloading on skeletal muscle mass and function in humans. J Appl Physiol 1991;70:1882-1885.

16. Desplanches D, Hoppeler H, Mayet MH, Denis C, Claassen H, Ferretti G. Effects of bedrest on deltoideus muscle morphology. Acta Physiol Scand 1998;162:135-140.

17. Kakurin LI, Lobachik VI, Mikhailov VM, Senkevich YA. Antiorthostatic hypokinesia as a method of weightlessness simulation. Aviat Space Environ Med 1976;47:1083-1086.

18. Kakurin LI, Kuzmin MP, Matsnev EI, Mikhailov VM. Physiological effects induced by antiorthostatic hypokinesia. Life Sci Space Res 1976;14:101-108.

19. Berg HE, Tedner B, Tesch PA. Changes in lower limb muscle crosssectional area and tissue fluid volume after transition from standing to supine. Acta Physiol Scand 1993;148:379-385. 
20. LeBlanc A, Evans H, Schonfeld E, Ford J, Schneider V, Jhingran S, et al. Changes in nuclear magnetic resonance (T2) relaxation of limb tissue with bed rest. Magn Reson Med 1987;4:487-492.

21. Rittweger J, Frost HM, Schiessl H, Ohshima H, Alkner B, Tesch P, et al. Muscle atrophy and bone loss after 90 days of bed rest and the effects of flywheel resistive exercise and pamidronate: results from the LTBR study. Bone 2005;36:1019-1029.

22. Watanabe Y, Ohshima H, Mizuno K, Sekiguchi C, Fukunaga M, Kohri K, et al. Intravenous pamidronate prevents femoral bone loss and renal stone formation during 90-day bed rest. J Bone Miner Res 2004;19:1771-1778.

23. Rittweger J, Felsenberg D, Maganaris CN, Ferretti JL. Vertical jump performance after 90 days bed rest with and without flywheel resistive exercise, including a 180 days follow-up. Eur J Appl Physiol 2007;100:427-436.

24. Berg HE, Tesch PA. A gravity-independent ergometer to be used for resistance training in space. Aviat Space Environ Med 1994;65: $752-756$.

25. Rittweger J, Beller G, Ehrig J, Jung C, Koch U, Ramolla J, et al. Bone-muscle strength indices for the human lower leg. Bone 2000; 27:319-326.

26. Zorbas YG, Yarullin VL, Denogratov SD, Deogenov VA. Fluid volume measurements in normal subjects to disclose body hydration during acute bed rest. Int Urol Nephrol 2003;35:457-465.

27. Zwart SR, Crawford GE, Gillman PL, Kala G, Rodgers AS, Rogers A, et al. Effects of 21 days of bed rest, with or without artificial gravity, on nutritional status of humans. J Appl Physiol 2009;107: 54-62.

28. Gluer CC, Blake G, Lu Y, Blunt BA, Jergas M, Genant HK. Accurate assessment of precision errors: how to measure the reproducibility of bone densitometry techniques. Osteoporos Int 1995;5:262-270.

29. Crawley MJ. The R book. Chichester, Sussex, UK: Wiley; 2007.

30. Wong AK, Bhargava A, Beattie K, Gordon CL, Pickard L, Webber CE. Muscle density, a surrogate of intramuscular adiposity derived from pQCT, is an independent correlate of fractures in women. J Bone Miner Res 2011;26(Suppl 1):FR0357.

31. Stryer L. Biochemie [Biochemistry]. Heidelberg: Spektrum Verlag; 1990.

32. Zorbas YG, Yarullin VL, Denogratov SD, Luzhkov YS, Kuznetsov NA. Fluid volume compartments and biochemical measurements for disclosing fluid depletion during acute and rigorous bed rest in normal subjects. International urology and nephrology 2002;34 $467-476$.

33. Mizuno M, Kimura Y, Iwakawa T, Oda K, Ishii K, Ishiwata K, et al Regional differences in blood volume and blood transit time in resting skeletal muscle. Jpn J Physiol 2003;53:467-470.

34. Hayashi Y, Ikata T, Takai H, Takata S, Sogabe T, Koga K. Time course of recovery from nerve injury in skeletal muscle: energy state and local circulation. J Appl Physiol 1997;82:732-737.

35. Degens H, Kosar SN, Hopman MT, de Haan A. The time course of denervation-induced changes is similar in soleus muscles of adult and old rats. Appl Physiol Nutr Metab 2008;33:299-308.

36. Colleran PN, Wilkerson MK, Bloomfield SA, Suva LJ, Turner RT, Delp MD. Alterations in skeletal perfusion with simulated microgravity: a possible mechanism for bone remodeling. J Appl Physiol 2000; 89:1046-1054.

37. Rittweger J, Beller G, Armbrecht G, Mulder E, Buehring B, Gast U, et al. Prevention of bone loss during 56 days of strict bed rest by side-alternating resistive vibration exercise. Bone 2010;46:137-147.

38. Hagberg S, Haljamae H, Rockert H. Shock reactions in skeletal muscle. 3. The electrolyte content of tissue fluid and blood plasma before and after induced hemorrhagic shock. Ann Surg 1968;168: 243-248.

39. Zange J, Muller K, Schuber M, Wackerhage H, Hoffmann U, Gunther RW, et al. Changes in calf muscle performance, energy metabolism, and muscle volume caused by long-term stay on space station MIR. Int J Sports Med 1997;18(Suppl 4):S308-S309.

40. Hoppeler H. Exercise-induced ultrastructural changes in skeletal muscle. Int J Sports Med 1986;7:187-204.

41. Hoppeler H, Howald H, Conley K, Lindstedt SL, Claassen H, Vock $\mathrm{P}$, et al. Endurance training in humans: aerobic capacity and structure of skeletal muscle. J Appl Physiol 1985;59:320-327.

42. Hoppeler H, Billeter R, Horvath PJ, Leddy JJ, Pendergast DR. Muscle structure with low- and high-fat diets in well-trained male runners. Int J Sports Med 1999;20:522-526.

43. Goodpaster BH, Theriault R, Watkins SC, Kelley DE. Intramuscular lipid content is increased in obesity and decreased by weight loss. Metabolism 2000;49:467-472. 